Journal of Computer Science 7 (2): 284-290, 2011

ISSN 1549-3636

(C) 2011 Science Publications

\title{
Performance Analysis of Multi-user Multi Input Multi Output- Interleave-Division Multiple-Access System Employing Turbo Coding with Multi-User Detection over Frequency-Selective Wireless Communication Channel
}

\author{
${ }^{1}$ Kuttathatti Srinivasan Vishvaksenan, ${ }^{2}$ R. Seshasayanan and ${ }^{3}$ Yuvaraj Krishnamoorthy \\ ${ }^{1}$ SSN College of Engineering, Chennai, India \\ ${ }^{2}$ Department of Electronics and Communication Engineering, \\ College of Engineering, guindy, Anna University, Chennai, India \\ ${ }^{3}$ Student of SSN College of Engineering, Chennai, India
}

\begin{abstract}
Problem statement: This study presents the performance analysis of multi-user Multi Input Multi Output (MIMO) assisted interleave based multiple-access system. In IDMA, different interleavers are used to distinguish users as against different signature sequence in a conventional code-Division Multiple-Access (CDMA) scheme. Approach: The basic principle of IDMA is that the interleaver is unique for the users. Results: In this study, we consider that Interleavers are generated independently and randomly. . Also the IDMA technique is extended to multi user MIMO IDMA with multi-user detection. At the receiver, OSIC detector is realized using ZF for frequency fading channel to combat MAI and MUI problem. The performance of the system is analyzed for different channel conditions using extensive simulation runs based on Monte Carlo simulation trials. Conclusion: It is shown that the IDMA scheme can achieve near single user performance in situations with very large numbers of users while maintaining very low receiver complexity. It is discerned from the computer simulation results that IDMA outperforms CDMA in frequency selective channel for high load conditions.
\end{abstract}

Key words: Multiple Antenna, Code Division Multiple Access (CDMA), International Telecommunication Union (ITU), channel model, channel capacity, iterative decoding, Multi-User Detection (MUD), Likelihood Ratio (LLR)

\section{INTRODUCTION}

Code division multiple access system is the most widely used system for multi-user communications. But the performance of CDMA (Yeo et al., 2003; Kuppusamy et al., 2008) is limited by multiple access interference and inter symbol interference. With CDMA fading is circumvented by the use of interleavers placed between FEC and spreading. After the invention of joint Turbo type receivers, extensive studies have been made to mitigate MAI and ISI (Telatar, 1999; Nagaradjane et al., 2009a; 2009b) employing joint detection and decoding. But high complexity of optimal detection precludes its implementation for signal detection. The interleaves, which are primarily implemented for combating fading in CDMA system, can be employed to distinguished user signals. This principle has been investigated (Ping et al., 2006; Schoeneich and Hoeher, 2004) and its potential merits have been addressed. This system in which interleavers are employed as means of user separation is called IDMA (Kusume et al., 2007) IDMA retains several advantages of CDMA like a asynchronous transmission, Cross cell interference and robustness against fading. In interleave division multiple access system, user specific interleavers are employed. IDMA (Sun et al., 2001; Wang et al., 2010) has been demonstrated to outperform CDMA with multi-user detection (Wang et al., 2010) Theoretical analysis has shown that optimal capacity of multiple access scheme can be achieved when the entire bandwidth is utilized for coding .In IDMA, user specific interleavers combine with low rate channel coding is used for user separation. Here the interleavers are generated randomly and independently. It is a wide band system and multi-user detection with moderate complexity can be used for signal detection.

Multi Input and Multi Output (MIMO) (Novak et al., 2007; Ershadi and Bakhshi, 2010; Sayadi et al., 2009; Nasseri and Bakhshi, 2010; Yahya et al., 2010; Yang et al., 2010; Zhou et al., 2010) system has proved in the recent past to provide very high capacity without

Corresponding Author: Kuttathatti Srinivasan Vishvaksenan, Department of Electronics and Communication Engineering, SSN College of Engineering, Chennai, India 
any increase in the transmission bandwidth and power. The information-theoretic capacity of these multipleinput multiple-output channels was shown to grow linearly with smaller numbers of transmit and receive antennas in rich scattering environments and at sufficiently high Signal-To-Noise (SNR) ratios. MIMO (Muthaiyah, 2004) profile in any wireless communication system can be realized by two schemes namely, (1) using the classical BELL labs architecture (VBLAST-Vertical Bell Laboratories Space Time architecture) and (2) Space Time Block Codes. So combining Vblast architecture with the IDMA system can result in MIMO-IDMA that can offer bandwidth efficiency, space multiplexing and lower speed parallel type of signal processing and interference rejection capability (ISI reduction) in high data-rate transmission. Of late, significant progress has been made in multiuser detection for code-division multiple-access (CDMA). Systems In this study, we investigate the performance of the MIMO assisted interleave division multiple access (MIMO- IDMA) scheme with multiuser detection. We will show that the MIMO IDMA scheme can provide better performance with the aid of VBLAST/ZF detection technique.

Also this approach is independent of the number of users and gives better performance in multipath environment. The rest of the study is organized as follows- the transmitter and receiver structures for CDMA and IDMA is presented in section II. System model is presented in section III. Section IV gives the essence of symbol detection techniques. Estimation technique is presented in Section V. Performance results and discussions are presented in section VI. Eventually conclusions are drawn in section VII.

Transmitter and receiver structures for CDMA AND IDMA: The data sequence of the $m$-th layer is denoted as $\mathrm{x}_{\mathrm{m}}$ and the corresponding encoded and interleaved sequence is denoted as $d_{m}$.

In Fig. 1, a DS-CDMA system is illustrated where the arrangement of interleaving $(\pi)$ and spreading $(C)$ is reversed. Now, different interleavers distinguish distinct data streams. This special case of DS-CDMA is called code-spread CDMA, chip-interleaved CDMA (cI-CDMA), or interleave-division multiple access (IDMA) in the literature.

The upper part of Fig. 2 shows the transmitter structure of the multiple access schemes under consideration with $\mathrm{K}$ simultaneous users and $\mathrm{V}$-Blast architecture. The input data sequence $\mathrm{x}_{\mathrm{k}}$ of user-k is encoded based on a low-rate code $\mathrm{S}$, generating a coded sequence $c_{k}=\left[c_{k}(1), \ldots, c_{k}(j), \ldots, c_{k}(J)\right]$, where $J$ is the frame length. The elements in ck are referred to as coded bits. Then ck is permutated by an interleaver pk, producing $d_{k}=\left[d_{k}(1), \ldots, d_{k}(j), \ldots, d_{k}(J)\right]$. Following

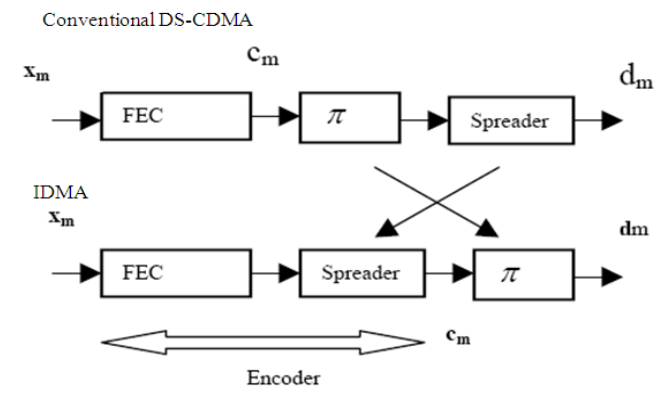

Fig. 1: Comparison of conventional DS-CDMA with IDMA

the CDMA convention, we call the elements in $d_{k}$ "chips". The interleavers solely distinguish users, hence the name Interleave-Division Multiple-Access (IDMA). In this study we consider a multi-user MIMO IDMA system. Each of the synchronous user is equipped with $M$ transmit antennas and they communicate to a single base station, equipped with $\mathrm{N}$ receive antennas. MIMO profile (Sun et al., 2011) is realized by using VBLAST (Vertical Bell Laboratories Space Time architecture).

In this contribution, we consider K-user up-link communication with each mobile station equipped with 2 transmitter antenna and the base station equipped with 2 receiver antenna . Let $\mathrm{Nt} X 2$ component transmitted vector of $\mathrm{k}^{\text {th }}$ user be denoted by $\mathrm{d}_{\mathrm{k}}=\left[\mathrm{d}_{1}(1), \ldots, \mathrm{d}_{2}(\mathrm{j})\right.$, $\left.\ldots, \mathrm{d}_{\mathrm{Nt}}\right]^{\mathrm{T}}$. This $\mathrm{dk}$ will be transmitted to $\mathrm{M}$ base station.

In this contribution, we assume that channel matrix that connects $\mathrm{K}_{\text {th }}$ user and $\mathrm{M}_{\text {th }}$ base station is frequency selective. The channel model assumed in our study is based on Rayleigh fading channel (Zhou et al., 2010) with 3 tap and Doppler shift $=10$ and ITU model with rich spatial scattering. The channel profile is detailed in Table 1.

With the help of the parameter defined in Table 1, the impulse response from ith transmitter antenna to jth receiver antenna (Brink, 2001) may be defined as:

$\mathrm{h}_{\mathrm{ji}}=\sum \mathrm{h}_{\mathrm{ji}}^{1} \partial\left(\mathrm{t}-\tau_{1}\right)$

Where:

$\mathrm{h}_{\mathrm{ji}}^{1}=$ Complex zero-mean Gaussian random process with variance

$\mathrm{p}\left(\tau_{1}\right), \mathrm{h}_{\mathrm{ji}}^{1}=$ Correlated with other paths and channels

$\mathrm{L}=$ Denotes the total number of paths between

$\mathrm{i}_{\text {th }}=$ Transmit

$\mathrm{j}_{\text {th }} \quad=$ Receive antenna

The $N_{r}$ length UL received vector $y$ at the $m_{t h}$ base station can be expressed as:

$\mathrm{y}=\sum_{\mathrm{k}=1}^{\mathrm{K}} \mathcal{H}_{\mathrm{k}} \mathrm{x}_{\mathrm{k}}+\mathrm{n}$ 
Table 1: Channel model parameter

\begin{tabular}{|c|c|c|c|c|c|c|}
\hline \multirow[t]{2}{*}{ Path Number } & \multicolumn{2}{|c|}{$\begin{array}{l}\text { Rayleigh fading } \\
\text { Channel }\end{array}$} & \multicolumn{2}{|c|}{$\begin{array}{l}\text { ITU-channnel-Pedestrain test } \\
\text { environment }\end{array}$} & \multicolumn{2}{|c|}{$\begin{array}{l}\text { ITU-channnel-Vehicular test } \\
\text { environment }\end{array}$} \\
\hline & Delay $(\mu \mathrm{s})$ & Power (dB) & Delay (ns) & Power (dB) & Delay (ns) & Power $(\mathrm{dB})$ \\
\hline$\overline{1}$ & 0.1 & 2 & 0 & 0 & 0 & -2.5 \\
\hline 2 & 0.2 & 3 & 200 & -0.9 & 300 & 0 \\
\hline \multirow[t]{4}{*}{3} & 0.3 & 4 & 800 & -4.9 & 8900 & -12.8 \\
\hline & & & 12000 & -8.0 & 12900 & -10.0 \\
\hline & & & 2300 & -7.8 & 17100 & -25.2 \\
\hline & & & 3700 & -23.9 & 20000 & -16.0 \\
\hline
\end{tabular}

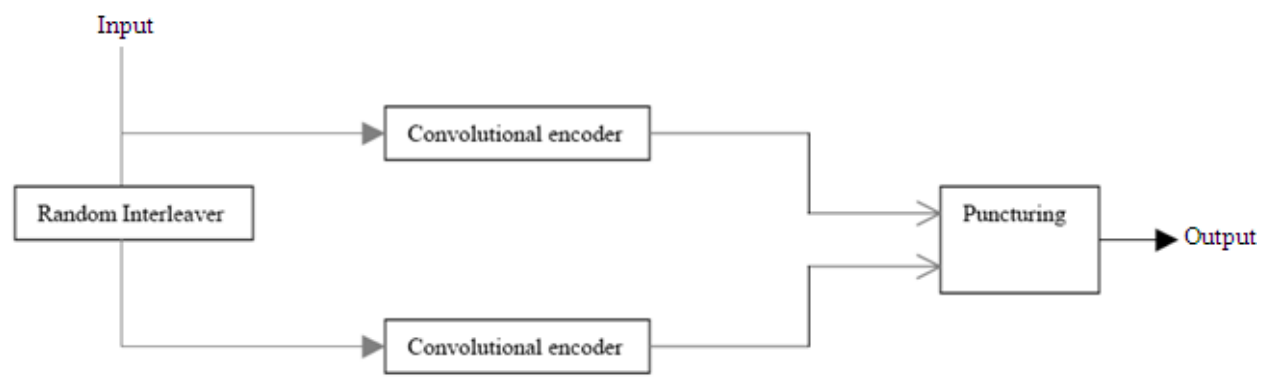

Fig. 2: Structure of turbo encoder

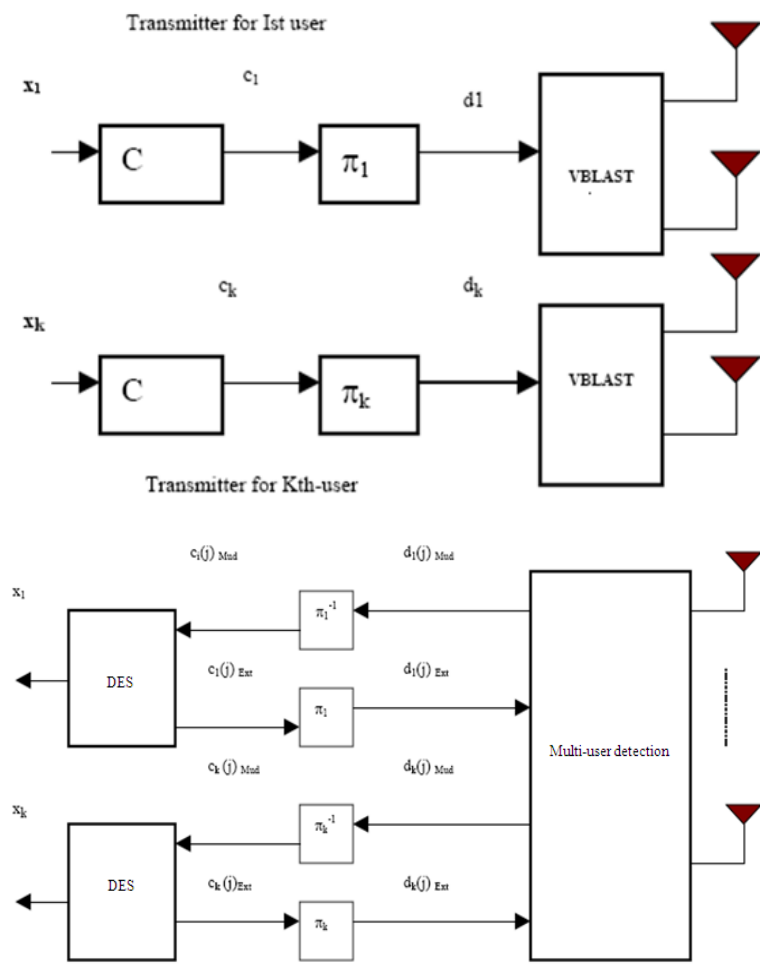

Fig. 3 Block diagram illustrating the principles of up link communication for IDMA system with Transmitter and (iterative) receiver structures and $\mathrm{K}$ simultaneous users and V-Blast architecture
$=\underbrace{\mathcal{H}_{\mathrm{k}} \mathcal{P}_{\mathrm{k}} \mathrm{d}_{\mathrm{k}}}+\underbrace{\sum_{\mathrm{i}=1, \mathrm{i} i \mathrm{k}}^{\mathrm{K}} \mathcal{H}_{\mathrm{k}} \mathcal{P}_{\mathrm{i}} \mathrm{d}_{\mathrm{i}}+\mathrm{n}, \mathrm{k}=1,2, \ldots, \mathrm{k}}$

Desired signal MUI

Symbol detection technique: Considering a synchronous signal over a time variant channel, the received signal at the time instant $\mathrm{j}$ is given as:

$r(j)=\sum_{k=1}^{K} h_{k} d_{k}(j)+n(j), j=1,2, . . J$

Where:

$\mathrm{H}_{\mathrm{k}} \quad=$ The channel co-efficient for user

$\mathrm{K},\{\mathrm{n}(\mathrm{j})\}=$ Samples of an AWGN process with

$\sigma^{2} \quad=$ voriance

Here perfect knowledge of the channel state information is assumed to be available at the receiver. In each use of the MIMO channel, a vector $d=\left(d_{1}, d_{2} \ldots\right.$ $\left.d_{M}\right)^{T}$ of complex numbers is sent and a vector $r=\left(r_{1}\right.$, $\left.r_{2}, \ldots \ldots, r_{N}\right)^{T}$ of complex numbers is received. We assume an input-output relationship of the form:

$\mathrm{r}=\mathrm{Hd}+\mathrm{n}$

Where: 
$\mathrm{H}=\mathrm{N} \times \mathrm{M}$ matrix representing the scattering effects of the channel

$\mathrm{n}=\left(\mathrm{n} 1, \mathrm{n}_{2}, \ldots \ldots, \mathrm{n}_{\mathrm{N}}\right)^{\mathrm{T}}=\quad$ Noise vector

Throughout, we assume that $\mathrm{H}$ is a random matrix with independent complex Gaussian elements $\left\{\mathrm{h}_{\mathrm{ij}}\right\}$ with mean 0 and unit variance, denoted by $\mathrm{h}_{\mathrm{ij}} \sim \mathrm{CN}(0 ; 1)$. We also assume throughout that $\mathrm{n}$ is identical and independent distributed (i.i.d) complex Gaussian random vector. The symbol detection problem considered in this study is the problem of estimating the MIMO channel input vector $\mathrm{x}$ given the received vector $r$ assuming that the receiver has perfect knowledge of $\mathrm{H}$. The decision is made on a symbol-by-symbol basis. The next section discusses the VBLAST detector employed in our study.

V-BLAST/ZF detection: For user-k, the corresponding ESE outputs $\left\{\mathrm{L}\left(\mathrm{d}_{\mathrm{k}}(\mathrm{j})\right), \mathrm{j}=1,2, \ldots . \mathrm{J}\right\}$ are de-interleaved to form $\left\{L\left(d_{k}(j)\right), j=1,2, \ldots . J\right\}$ and delivered to the DES for user-k. The DES performs a soft-in/soft-out chip-bychip de-spreading operation as detailed below. For simplicity, we focus on the chips related to $\mathrm{d} 1(\mathrm{k})$, the first bit of user-k. The treatment for other chips is similar.

The V-BLAST detection algorithm Clemens Novak (2007) is a recursive procedure that extracts the components of the transmitted vector $d$ according to a certain ordering $\left(\mathrm{k}_{1}, \mathrm{k}_{2} \ldots \mathrm{k}_{\mathrm{M}}\right)$ of the elements of $\mathrm{d}$, where, $\left(\mathrm{k}_{1}, \mathrm{k}_{2} \ldots \mathrm{k}_{\mathrm{M}}\right)$ is a permutation of $(1 \ldots \mathrm{M})$. In VBLAST, this permutation depends on $\mathrm{H}$ (which is known at the receiver by assumption) but not on the received vector $r$. The VBLAST/ZF algorithm is a variant of VBLAST derived from $Z F$ rule. The algorithm determines the order of layers to be detected performs nulling and computes the decision statistics. It then slices the computed decision statistics and yields the decision by performing cancellation with the help of decision feedback and finally computes the new pseudo- inverse for the next iteration. V-BLAST/ZF may be seen as a successive-cancellation scheme derived from the $\mathrm{ZF}$ scheme:

$$
\begin{aligned}
& \mathrm{W}_{1}=\mathrm{H}^{+} \\
& \mathrm{i}=1
\end{aligned}
$$

where, $\mathrm{W}$ is the weight matrix that depends on the channel fading $\mathrm{H}$.

\section{Recursion:}

$$
\begin{aligned}
& \mathrm{k}_{\mathrm{i}}=\underset{j \notin\left\{\mathrm{k}_{1} \ldots . \mathrm{k}_{\mathrm{i}-1}\right\}}{\arg \max }\left\|\left(\mathrm{W}_{\mathrm{i}}\right)_{\mathrm{j}}\right\| \\
& \mathrm{Y}_{\mathrm{k}_{\mathrm{i}}}=\left(\mathrm{W}_{\mathrm{i}}\right)_{\mathrm{k}_{\mathrm{i}}} \mathrm{r}_{\mathrm{i}} \\
& \hat{\mathrm{a}}_{\mathrm{k}_{\mathrm{i}}}=\mathrm{Q}\left(\mathrm{Y}_{\mathrm{k}_{\mathrm{i}}}\right) \\
& \mathrm{r}_{\mathrm{i}+1}=\mathrm{r}_{\mathrm{i}}-\hat{\mathrm{a}}_{\mathrm{k}_{\mathrm{i}}}(\mathrm{H})_{\mathrm{k}_{\mathrm{i}}} \\
& \mathrm{W}_{\mathrm{i}+1}=\mathrm{H}_{\mathrm{k}_{\mathrm{i}}}^{+} \\
& \mathrm{i}=\mathrm{i}+1
\end{aligned}
$$

where. $\mathrm{H}^{+}$is the Moore-Penrose pseudo-inverse of $\mathrm{H}$, the channel matrix. $\left(\mathrm{W}_{\mathrm{i}}\right)_{\mathrm{j}}$ is the jth row of $\mathrm{W}_{\mathrm{i}} \mathrm{Q}($.$) is a$ quantizer to the nearest signal point, $(\mathrm{H})_{\mathrm{kj}}$ denotes the $\mathrm{k}_{\mathrm{i}}$ th column of $\mathrm{H}$. $\mathrm{H} \pm_{\mathrm{kj}}$ denotes the matrix obtained by zeroing the columns $\mathrm{k}_{1}, \mathrm{k}_{2}, \ldots . \mathrm{k}_{\mathrm{i}}$ of $\mathrm{H}$.. In the above algorithm, Eq. 7 determines the order of the channels to be detected. This is done by choosing the channels with the smallest noise variance. Using Eq. 8 the corresponding layer is decided based on the smallest noise variance and quantization is performed with the help of the Eq. 8. In Eq. 9, Interference mitigation is performed through decision feedback. Equation 10 calculates the weight matrix for the next iteration. The $\mathrm{ZF}$ creates set of sub-channels by forming The ZF creates set of sub-channels by forming:

$\hat{\mathrm{a}}_{\mathrm{zf}}=\left(\mathrm{H}^{+} \mathrm{H}\right) \mathrm{a}+\mathrm{H}^{+} \mathrm{v}$

The order selection rule prioritizes the sub-channel with the smallest noise variance.

Chip-by-chip detection: The a posteriori LLR (Ping et al., 2006) for $\mathrm{x}_{\mathrm{k}}(\mathrm{j})$ can be computed using $\left\{\mathrm{L}_{\mathrm{k}}(\mathrm{c}(\mathrm{j}))\right\}$ as:

$$
\begin{aligned}
L\left(x_{k}(j)\right) & \equiv \log \left[\frac{\operatorname{Pr}\left(x_{k}(j)=+1 \mid r\right)}{\operatorname{Pr}\left(x_{k}(j)=-1 \mid r\right)}\right] \\
& =\sum_{j=1}^{s} s_{k}(j) L\left(c_{k}(j)\right)
\end{aligned}
$$

The extrinsic LLR for a chip cj(k) within $\mathrm{d} 1(\mathrm{k}) \mathrm{s}(\mathrm{k})$ is defined by:

$$
\left(c_{k}(j)\right)_{\text {Ext }} \equiv \log \left[\frac{\operatorname{Pr}\left(c_{k}(j)=+1 \mid r\right)}{\operatorname{Pr}\left(c_{k}(j)=-1 \mid r\right)}\right]-L\left(c_{k}(j)\right)
$$


The extrinsic LLRs $\left\{\left(\mathrm{c}_{\mathrm{k}}(\mathrm{j})_{\mathrm{Ext}}\right)\right\}$ form the outputs of the DES and are fed back to the ESE after interleaving. In the next iteration, $\left\{\right.$ Ext $\left.\left(\mathrm{d}_{\mathrm{k}}(\mathrm{j})\right)\right\}$ are used to update $\left\{\mathrm{E}\left(\mathrm{d}_{\mathrm{k}}(\mathrm{j})\right)\right\}$ and $\left.\left\{\operatorname{var~}_{\mathrm{k}}(\mathrm{j})\right)\right\}$ as:

$$
\begin{aligned}
& E\left(d_{k}(j)\right)=\frac{\exp \left(\left(d_{k}(j)\right)_{\text {Ext }}\right)-1}{\exp \left(\operatorname{Ext}\left(d_{k}(j)\right)\right)+1}=\tanh \left[\frac{\left(d_{k}(j)\right)_{\text {Ext }}}{2}\right] \\
& \operatorname{Var}\left(d_{k}(j)\right)=1-E\left(d_{k}(j)\right)^{2}
\end{aligned}
$$

The iteration is carried out till variance $=0$ and mean $=1$

\section{RESULTS AND DISCUSSION}

In this section we present the simulation results of our analysis. Table 1 summarizes the simulation parameters.

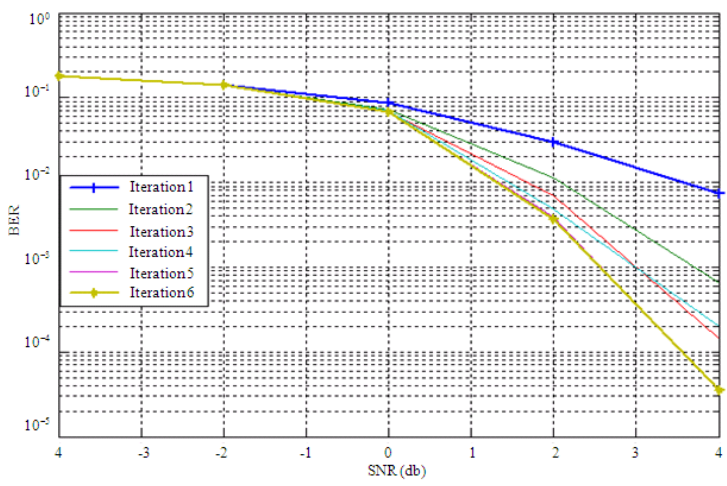

Fig. 4: Bit Error Rate (BER) performance of turbo coded MIMO-IDMA with ZF for 50 users over Rayleigh fading channel

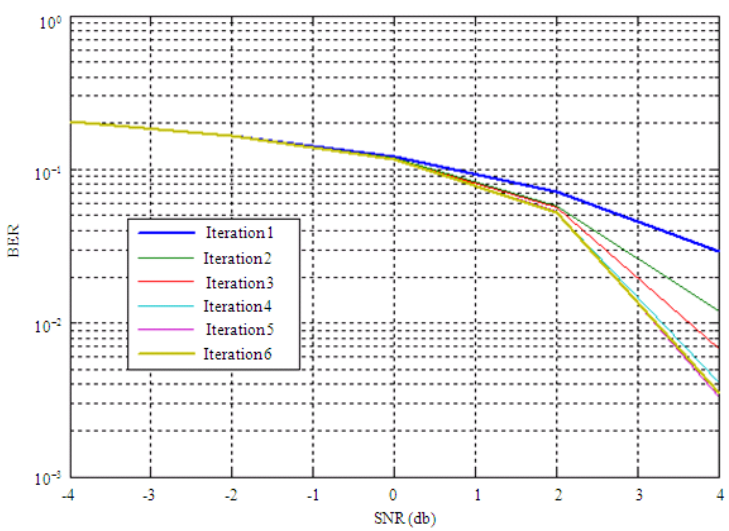

Fig. 5: Bit Error Rate (BER) performance of turbo coded MIMO-IDMA with ZF for 50 users over correlated Rayleigh fading channel
Figure 4 shows Bit Error Rate (BER) performance of turbo coded MIMO-IDMA with ZF for 50 users over frequency selective Rayleigh fading channel. Figure 5 expounds Bit error rate (BER) performance of turbo coded MIMO-IDMA with ZF for 50 users over correlated frequency selective Rayleigh fading channel. Figure 6 evinces the comparison of Bit Error Rate (BER) performance of turbo coded MIMOIDMA with ZF over correlated and uncorrelated frequency selective Rayleigh fading channel for 50 users. Figure 7 Bit Error Rate (BER) performance of turbo coded MIMO-IDMA with ZF for ITU-pedestrian test environment channel model. Figure 8 Bit Error Rate (BER) performance of turbo coded MIMO-IDMA with ZF for ITU-vehicular test environment channel model.

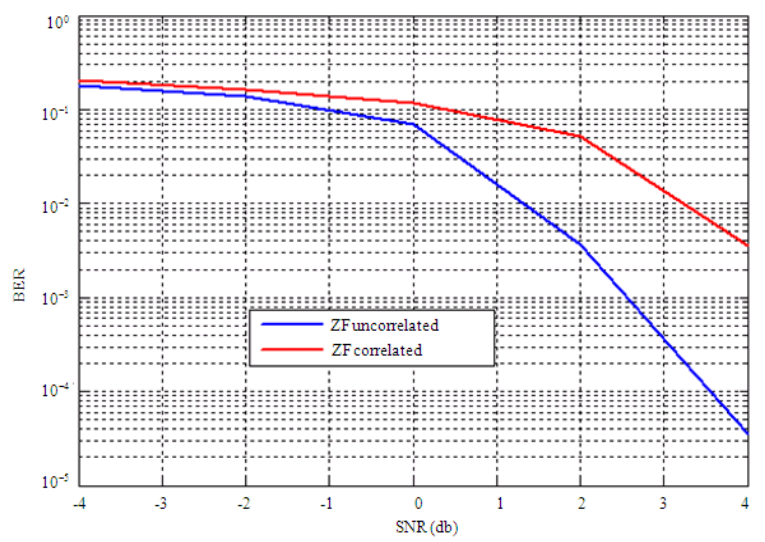

Fig. 6: Bit Error Rate (BER) performance of turbo coded MIMO-IDMA with ZF over correlated and uncorrelated channel for 50 users

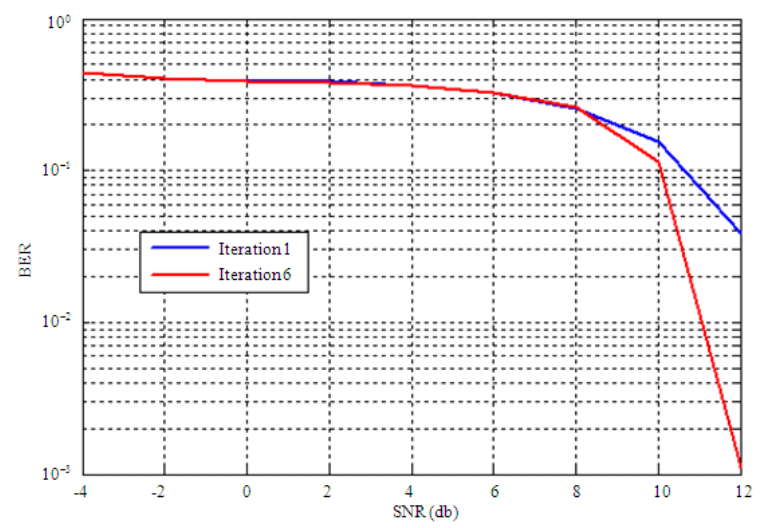

Fig.7: Bit Error Rate (BER) performance of turbo coded MIMO-IDMA with ZF for ITUpedestrian test environment channel model 


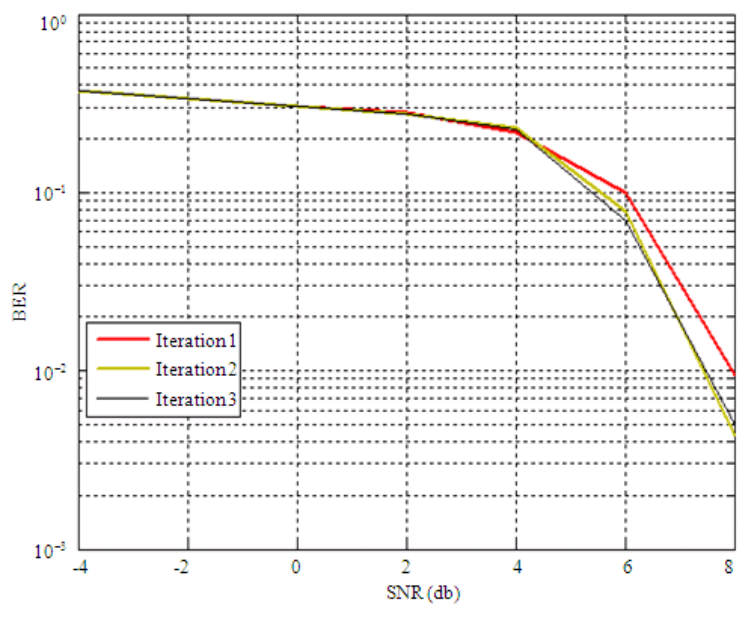

Fig. 8: Bit error rate (BER) performance of turbo coded MIMO-IDMA with ZF for ITU-vehicular test environment channel model

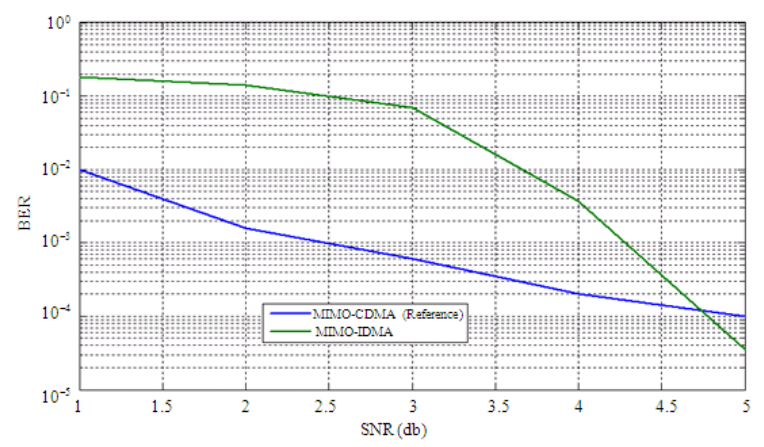

Fig. 9: Bit Error Rate (BER) performance of turbo coded MIMO-CDMA and MIMO-IDMA with ZF detector over Rayleigh fading channel

Figure 9 elucidate the comparison of Bit Error Rate (BER) performance of turbo coded MIMO-CDMA and MIMO-IDMA with ZF detector over frequency selective Rayleigh fading channel. From our analysis, it can be seen that MIMO-IDMA with ZF results in better interference suppression. Also it can be concluded that MIMO assisted IDMA outperforms CDMA based on multi-user MIMO system in terms of achievable error rate for frequency selective fading channel

\section{CONCLUSION}

In this study, we analyze the performance of MIMO assisted IDMA system using OSIC detector supports MUD. It is discerned from the analysis that multipath can severely degrade the achievable BER. Also MUI can result in further degradation in the system performance. It is shown through computer simulation that in the context of multi-user scenario, MIMO-IDMA system with MUD can mitigate multiuser interference, thereby achieving higher capacity in terms of supporting more number of users and hence it is interesting topic for the future research.

\section{REFERENCES}

Brink, S.T., 2001. Convergence behavior of iteratively decoded parallel concatenated codes. IEEE Trans. Commun., 49: 1727-1737. DOI: 10.1109/26.957394

Ershadi, E. and H. Bakhshi, 2010. A preamble-based frequency offset compensation scheme in multiple input multiple output orthogonal frequency division multiplexing systems. J. Comput. Sci., 6: 478-483. DOI: 10.3844 jessp.2010.478.483

Kuppusamy, M., M. Pahlavani and A.S. Saleh, 2008. Fostering ICT development for growth: measuring the payoffs for australia and the asean-5 countries. Am. J. Applied Sci., 5: 1676-1685.DOI: 10.3844/ajassp.2008.1676.1685

Kusume,K., G. Dietl, W. Utschick and G. Bauch, 2007. Performance of interleave division multiple access based on minimum mean square error detection. Proceeding of the IEEE International Conference on Communications, June 24-28, Glasgow, pp: 2961-2966. DOI: 10.1109/ICC.2007.492

Muthaiyah, S., 2004. Key success factors of $3^{\text {rd }}$ generation mobile network services for $\mathrm{m}$ commerce in Malaysia. Am. J. Applied Sci., 1: 261-265. DOI: 10.3844/ajassp.2004.261.265

Nagaradjane, P., A.S.S. Vasan, L. Krishnan and A. Venkataswamy, 2009b. Joint VBLAST/STBC assisted MC DS/CDMA system with multiuser detection. Proceeding of the 5th International Conference on Wireless Communications, Networking and Mobile Computing, Sept. 24-26, Beijing, pp: 1-4. DOI: 10.1109/WICOM.2009.5301618

Nagaradjane, P., A.S.S. Vasan and L. Krishnan, 2009a. A robust space time co-channel interference mitigation and detection technique for multiuser MIMO multicarrier DS/CDMA systems. Proceeding of the 1st IEEE International Conference on Wireless Communication Vehicular Technology, Information Theory, Aerospace and Electronic Systems Technology, May 17-20, Aalborg, $\quad$ pp: 842-846. DOI: 10.1109/WIRELESSVITAE.2009.5172559

Nasseri, M. and H. Bakhshi, 2010. Iterative channel estimation algorithm in multiple input multiple output orthogonal frequency division multiplexing systems. J. Comput. Sci., 6: 224-228. DOI: $10.3844 /$ jcssp. 2010.224 .228 
Novak, C., F. Hlawatch and G. Matz, 2007. MIMOIDMA: Uplink multiuser MIMO communications using interleave-division multiple access and low complexity iterative receivers. Proceeding of the IEEE International Conference on in Acoustics, Speech and Signal Processing, (ICASSP'07), Honolulu, Hawaii, pp: 225-228.

Ping, L., L. Liu, K. Wu and W.K. Leung, 2006. Interleave division multiple-access. IEEE Trans. Wireless Commun. 5: 938-947. DOI: 10.1109/TWC.2006.1618943

Sayadi, F., M. Ismail, N. Misran, K. Jumari and M. Abdullah, 2009. Efficient detection algorithm for a multiple-input and multiple-output multiuser multicarrier code division multiple access in timevarying channels. Am. J. Eng. Applied Sci., 2: 635-642. DOI: 10.3844/ajeassp.2009.635.642

Schoeneich, H. and P.A. Hoeher, 2004. Adaptive interleave-divisionmultiple access-a potential air interface for $4 \mathrm{G}$ bearer services and wireless LANs. Proceeding of the WOCN, (WOCN'04), Muscat, Oman, pp: 179-182.

Sun, L., Z. Ye and Z. Zhang, 2011. Base station coordinated multi-user detection in multi-cell MIMO cellular systems. Inform. Technol. J., 10: 134-139. DOI: 10.3923/itj.2011.134.139
Telatar, I.E., 1999. Capacity of multi-antenna gaussian channels. Eur. Trans. Tel., 10: 585-595. DOI: $10.1002 /$ ett.4460100604

Wang, K.H., C.Q. Zhang and J.L. Xu, 2010. Comparison of user selection methods for multiuser MIMO-OFDM downlink with limited feedback. Inform. Technol. J., 9: 720-729. DOI: 10.3923/itj.2010.720.729

Yahya, A., F. Ghani, R. Badlishah and R. Malook, 2010. An overview of low-density parity checks codes. J. Applied Sci., 10: 1910-1915.

Yang, X.L., Y.M. Chen and B. Zhou, 2010. Constructing error-correcting codes for wireless sensor networks. Inform. Technol. J., 9: 1758-1760.

Yeo, E., B. Nikolic and V. Anantharam, 2003. Iterative decoder architectures. IEEE Commun. Magazine, 41: 132-140. DOI: 10.1109/MCOM.2003.1222729

Zhou, X., Y. Yang, H. Shan and Z. Wang, 2010. Performance study of a network coded nonorthogonal user cooperation system over nakagami-m channels. Inform. Technol. J., 9: 1353-1360. 